\title{
Diagnostic performance of digital tomosynthesis to evaluate silicone airway stents and related complications
}

\author{
Bo-Guen Kim ${ }^{1}$, Myung Jin Chung ${ }^{2}$, Byeong-Ho Jeong ${ }^{1}$, Hojoong Kim ${ }^{1}$ \\ ${ }^{1}$ Division of Pulmonary and Critical Care Medicine, Department of Medicine, Samsung Medical Center, Sungkyunkwan University School \\ of Medicine, Seoul, Republic of Korea; ${ }^{2}$ Department of Radiology and Center for Imaging Science, Samsung Medical Center, Sungkyunkwan \\ University School of Medicine, Seoul, Republic of Korea \\ Contributions: (I) Conception and design: BH Jeong, H Kim; (II) Administrative support: H Kim; (III) Provision of study materials or patients: BH \\ Jeong, H Kim; (IV) Collection and assembly of data: BG Kim, MJ Chung, BH Jeong; (V) Data analysis and interpretation: BG Kim, BH Jeong; (VI) \\ Manuscript writing: All authors; (VII) Final approval of manuscript: All authors. \\ Correspondence to: Hojoong Kim, MD, PhD. Division of Pulmonary and Critical Care Medicine, Department of Medicine, Samsung Medical Center, \\ Sungkyunkwan University School of Medicine, 81 Irwon-ro, Gangnam-gu, Seoul 06351, South Korea. Email: hjk3425@skku.edu.
}

Background: Digital tomosynthesis (DTS) is an imaging technique with benefits in reconstructing sequential cross-sectional images. We evaluated the diagnostic performance of DTS for silicone airway stents and stent-related complications in patients who underwent bronchoscopic intervention.

Methods: This retrospective study included patients who underwent bronchoscopic intervention after chest radiography (CXR) and DTS examinations from September 2013 to August 2020. The interval between CXR, DTS, and bronchoscopic intervention was a maximum of 10 days. CXR and DTS images were evaluated using a bronchoscopic view as a reference. We calculated the sensitivity, specificity, accuracy, positive predictive value, and negative predictive value for assessing the diagnostic performance.

Results: The total CXR, DTS, and bronchoscopic intervention-matching datasets comprised 213 cases from 119 patients and, silicone stents were present in 167 of them. The ability of DTS to detect silicone stents was better than that of CXR (sensitivity, $92.8 \%$ vs. $71.3 \%, \mathrm{P}<0.001$ ). Of the 167 cases with silicone stents, 53 experienced stent migration and 121 experienced stent obstructions due to granulation tissue or fibrosis. The sensitivity for detecting stent migration was also higher with DTS than with CXR (45.3\% vs. $24.5 \%, \mathrm{P}=0.025)$. The sensitivity for detecting the stent obstruction was better with DTS than with CXR (64.5\% vs. $19.0 \%, \mathrm{P}<0.001)$.

Conclusions: DTS was more sensitive and accurate in revealing silicone airway stents and silicone stentrelated complications than CXR. However, there were limitations in confirming stent migration and obstruction with DTS due to granulation tissue growth and fibrosis.

Keywords: Digital tomosynthesis (DTS); bronchoscopic intervention; silicone airway stent

Submitted Jun 21, 2021. Accepted for publication Sep 03, 2021.

doi: $10.21037 /$ jtd-21-1032

View this article at: https://dx.doi.org/10.21037/jtd-21-1032

\section{Introduction}

Plain chest radiography (CXR) has been used for diagnosing pulmonary diseases for a long time, and it remains a major diagnostic and screening tool (1). CXR examination has several advantages, such as low cost and short examination time, but also has low sensitivity in diagnosing diseases (2).
Chest computed tomography (CT) is highly sensitive for the diagnosis of pulmonary diseases $(3,4)$. However, the cost of CT is higher than that of CXR, and the radiation exposure to the patient is also relatively higher with CT. Digital tomosynthesis (DTS) is an imaging modality in which several individual projection images are acquired 
and reconstructed over a limited angular range (5). In the DTS, the tube performs a caudo-cranial sweep $\pm 15^{\circ}$ around the standard PA plane, collecting around 60 low-dose projections for 10-12 seconds (5). The angular movement of the tube enables the separation of overlapping anatomy in the DTS, but not in the CXR (Figure S1). DTS improves the visibility of thoracic structures as compared with CXR (6). Simultaneously, the examination can be conducted with a lower radiation dose than that required for chest CT (7-9). With this advantage, several studies have reported the diagnostic performance of DTS for pulmonary diseases, including lung nodules, mycobacterial infection disease, and lung cancer (10-14).

In patients with central airway stenosis, airway stents are often inserted to maintain airway patency. Occasionally, stent-related complications such as stent migration, granulation tissue growth, or fibrosis at the end of stents occur (15). Therefore, regular surveillance with imaging tests is needed for patients who have undergone silicone airway stent placement via bronchoscopic intervention. However, the silicone airway stent is radiolucent and difficult to visualize on a CXR. For this reason, our institution used DTS to examine patients who underwent bronchoscopic intervention. To the best of our knowledge, there have been no studies on the diagnostic performance of DTS for silicone airway stents and related complications. Therefore, we evaluated the diagnostic performance of DTS for silicone airway stents in patients who underwent bronchoscopic intervention.

We present the following article in accordance with the TREND reporting checklist (available at https://dx.doi. org/10.21037/jtd-21-1032).

\section{Methods}

\section{Patients}

Patients who underwent bronchoscopic intervention after CXR and DTS examinations at Samsung Medical Center between September 2013 and August 2020 were included in this study. The interval between CXR, DTS, and bronchoscopic intervention was established for a maximum of 10 days. In the case of repeated procedures in one patient, all procedures with CXR and DTS were included in this study. Patients whose intervals between examination and procedure exceeded 10 days or patients who did not have any of the above examinations were excluded from this study.

This study was approved by the Samsung Medical Center Institutional Review Board (IRB no. 2020-12-067), and informed consent was waived as we only used de-identified patients' information, and this study was a retrospective study. The study was conducted in accordance with the Declaration of Helsinki (as revised in 2013).

\section{CXR, DTS, and bronchoscopic intervention}

CXR and DTS were conducted on the same day under the same conditions. CXR examinations were performed using a cesium iodide/amorphous silicon flat-panel detector digital radiography system (Definium 8000; GE Healthcare, Chalfont St Giles, England), and all images were acquired in posteroanterior views at a tube voltage of $120 \mathrm{kVp}$ with automatic exposure control at a speed equivalent of 400 (11). After obtaining a scout image using CXR, all patients underwent DTS. DTS was conducted using a commercially available unit (Volume RAD; GE Healthcare) with a cesium iodide/amorphous silicon flat-panel detector system. Within approximately $10 \mathrm{~s}, 60$ low-dose projection images were acquired using a tube voltage of $100 \mathrm{kVp}$, a $1: 5$ dose ratio, and a $0.3-\mathrm{mm}$ additional copper filter. The detector was held in position, whereas the $\mathrm{X}$-ray tube was continuously subjected to vertical movement, from $-17.5^{\circ}$ to $+17.5^{\circ}$, around the standard orthogonal posteroanterior position. Finally, 60 projection images were obtained at the tube angle between $-15^{\circ}$ and $+15^{\circ}$; these were used to reconstruct approximately 54 coronal images with a nominal thickness of $4 \mathrm{~mm}$ without overlap $(11,16)$. The image was followed from the front skin to the back of the chest.

The bronchoscopic intervention was performed according to standard techniques $(17,18)$. Under general anesthesia, the patient was intubated with a rigid bronchoscope tube (Bryan Co., Woburn, MA, USA or KarlStorz, Tuttlingen, Germany). Then, a flexible bronchoscope (EVIS BF 1T260; Olympus Co., Tokyo, Japan) was passed through the rigid bronchoscope tube, and the central airway was evaluated. The airway stent used in the procedure was a silicone stent (Dumon stent, Novatech, La Ciotat, France) or natural stent (M1S, Seoul, Korea). For some patients, an "angulated stent" was inserted when the straight tube stent did not fit well into the tortuous or distal airway. This stent was created by cutting a straight conventional stent into two separate sections at an oblique angle and reattaching it at the desired angle using non-absorbable sutures (19). 


\section{Data collection}

The following data were obtained from the database: baseline characteristics such as age; sex; etiology of airway abnormality; and all CXR, DTS, and bronchoscopic images (obtained during the bronchoscopic intervention). A chest radiologist (MJC) and two pulmonologists (BGK and $\mathrm{BHJ})$ worked independently and analyzed the image data separately in a blinded manner. After that, the value from two or more of the three readings that showed the same result was selected as the final result. Consensus results of image data were evaluated using the bronchoscopic view as a reference for assessing diagnostic performance. The central airway was divided into three parts: trachea, right main bronchus (RMB), right bronchus intermedius (RBI), and left main bronchus (LMB). In the case of patients with airway stents, we evaluated the presence of the stent and whether the stent was angulated. To evaluate the diagnostic performance of stent-related complications, we assessed the presence of stent migration, stent obstruction due to granulation tissue, or fibrosis at the end of the stent.

\section{Statistical analysis}

Data are presented as number (\%) for categorical variables and median [interquartile range (IQR)] for continuous variables. All cases included in this study were evaluated as "Yes (Detected)" or "No (Not detected)" for each evaluation item (presence of a stent, stent angulation, stent migration, and stent obstruction). For example, if stent migration, stent obstruction, and stent angulation were not detected because the stent was not visible in the imaging examination, the test result was classified as "No (Not detected)." Based on this evaluation, a $2 \times 2$ contingency table was created. From these tables, we calculated the sensitivity, specificity, accuracy, positive predictive value (PPV), and negative predictive value (NPV) with $95 \%$ confidence interval (CI) for assessing the diagnostic performance of the modalities (20). To calculate the CIs for sensitivity, specificity, and accuracy, the Clopper-Pearson exact method was used. The CIs for the predictive values were standard logit CIs (21). The "N1 " Chi-squared test was used for comparison of proportions (22,23). Statistical significance was set at $\mathrm{P}<0.05$. All statistical analyses were performed using the SPSS software (IBM SPSS Statistics ver. 27, Chicago, IL, USA).

\section{Results}

A total of 119 patients were included in the study. The median age was 52 (IQR, 38-59) years, and $42 \%$ of the patients were male. The most common etiology was posttuberculosis bronchial stenosis $(64,53.8 \%)$, followed by post-intubation tracheal stenosis $(23,19.3 \%)$, malignant central airway obstruction $(11,9.2 \%)$, post-tracheostomy tracheal stenosis $(7,5.9 \%)$, relapsing polychondritis (4, 3.4\%), postoperative tracheobronchial stenosis $(3,2.5 \%)$, and post-radiation tracheobronchial stenosis (3, 2.5\%). The median interval between CXR, DTS, and bronchoscopic intervention was 5 days (IQR, 3-6 days). Further, 47 (39.5\%) patients had lesions on the trachea, $22(18.5 \%)$ patients, on the RMB \& RBI, and 49 (41.2\%) patients, on the LMB. Of the patients, $83(69.7 \%)$ had a silicone airway stent placed.

A total of $56(47.1 \%)$ patients underwent two or more bronchoscopic interventions. Finally, there were 213 CXR, DTS, and bronchoscopic intervention-matching datasets from 119 patients (Table 1).

\section{Diagnostic performance of DTS in the detection of silicone stent}

Of the 213 cases, silicone stents were present in 167 (78.4\%), and among them, the stents were angulated in 39 (39/213, 18.3\%) cases. The sensitivity of DTS in detecting the silicone stents was $92.8 \%$ (95\% CI, 87.8-96.2\%), specificity was $97.8 \%$ (95\% CI, 88.5-99.9\%), and accuracy was $93.9 \%$ (95\% CI, 89.9-96.7\%); the PPV was $99.4 \%$ (95\% CI, 95.7-99.9\%) and NPV was 79.0\% (95\% CI, 68.5-86.6\%). The sensitivity (92.8\% vs. $71.3 \%, \mathrm{P}<0.001)$, accuracy (93.9\% vs. $77.5 \%, \mathrm{P}<0.001)$, and NPV $(79.0 \%$ vs. $48.9 \%, \mathrm{P}<0.001)$ of DTS were significantly better than those of CXR (Table 2). The ability of DTS to detect stent angulation was also better than that of CXR. The sensitivity (66.7\% vs. $25.6 \%, \mathrm{P}<0.001)$ and NPV $(92.8 \%$ vs. $85.6 \%, \mathrm{P}=0.025)$ of DTS were significantly better than those of CXR (Table S1). Occasionally, DTS indicated the presence of a stent in places where the stent was actually absent. This occurred when a thick airway wall mimicked the stent in a location where the stent was previously placed (Figure S2).

There were differences in stent detection ability depending on the location: trachea, RMB and RBI, and LMB. In the LMB, DTS had the best ability to detect stents, with a sensitivity of $98.7 \%$ (95\% CI, 93.1-100.0\%). The sensitivity of DTS in detecting stents located in the trachea was the lowest at $85.9 \%$ (95\% CI, 75.0-93.4\%) (Table 2, Figure 1A). 
Table 1 Baseline characteristics $(\mathrm{N}=119)$

\begin{tabular}{lc}
\hline Variables & Data \\
\hline Age, years & $52(38-59)$ \\
Sex, male & $42(35.3)$ \\
Etiology & \\
PTBS & $64(53.8)$ \\
PITS & $23(19.3)$ \\
MCAO & $11(9.2)$ \\
PTTS & $7(5.9)$ \\
Relapsing polychondritis & $4(3.4)$ \\
POTS & $3(2.5)$ \\
PRTS & $3(2.5)$ \\
Others & $4(3.4)$ \\
Interval between CXR, DTS and & $5(3-6)$ \\
bronchoscopic intervention, days & \\
Location of airway lesions & \\
Trachea &
\end{tabular}

Data are presented as $\mathrm{n}(\%)$ or median (interquartile range). *, airway burn $(n=1)$, fistula $(n=1)$, idiopathic tracheal stenosis $(n=1)$, trauma $(n=1) ;^{\dagger}$, patients with one or more airway lesions; ${ }^{\ddagger}$, total of 221 cases of CXR, tomosynthesis, and rigid bronchoscopy-matching datasets from 123 patients. PTBS, post-tuberculosis bronchial stenosis; PITS, postintubation tracheal stenosis; MCAO, malignant central airway obstruction; PTTS, post-tracheostomy tracheal stenosis; POTS, postoperative tracheobronchial stenosis; PRTS, post-radiation tracheobronchial stenosis; CXR, chest radiography; DTS, digital tomosynthesis; $\mathrm{RMB}$, right main bronchus; $\mathrm{RBI}$, right bronchus intermedius; LMB, left main bronchus.

\section{Diagnostic performance for the detection of silicone stent- related complications}

Of the 213 cases, $53(53 / 213,24.9 \%)$ showed stent migration. The ability of DTS to detect stent migration was higher than that of CXR. For stent migration, the sensitivities of DTS and CXR was $45.3 \%$ (95\% CI, $31.6-59.6 \%)$ and $24.5 \%$ (95\% CI, $13.8-38.3 \%)(\mathrm{P}=0.025)$
(Figure 1B). The accuracies of DTS and CXR were $84.0 \%$ (95\% CI, 78.4-88.7\%) and 80.8\% (74.8-85.8\%) $(\mathrm{P}=0.396)$.

Of the total 213 cases, $121(121 / 213,56.8 \%)$ cases of stent obstruction were caused by granulation tissue or fibrosis. The ability of DTS to detect granulation tissue or fibrosis at the end of the stent was significantly better than that of CXR (Figure 1B). The sensitivity of DTS was $64.5 \%$ (95\% CI, $55.3-73.0 \%$ ), specificity was $85.9 \%$ (95\% CI, $77.1-92.3 \%$ ), and accuracy was $73.7 \%$ (95\% CI, $67.3-$ $79.5 \%$ ). For CXR, the sensitivity was $19.0 \%$ (95\% CI, 12.5 $27.1 \%$ ), specificity was $100.0 \%$ (95\% CI, 96.1-100.0\%), and accuracy was $54.0 \%$ (95\% CI, 47.1-60.8\%) (Table 3). As shown in Figure 2, granulation tissue grew on the distal end of the LMB stent and caused a partial obstruction. As shown in Figure 3, fibrosis occurred at the end of the midtracheal stent, creating an airway obstruction.

All true positive, false positive, true negative, and false negative values obtained to calculate diagnostic performance are summarized in Table S2 as a $2 \times 2$ contingency table.

\section{Discussion}

In this study, DTS was more capable of detecting silicone airway stents than CXR and showed high diagnostic performance. In particular, the stent located in the LMB was detected well. DTS also had a better ability to detect stent migration and stent obstruction due to granulation tissue or fibrosis than CXR. However, the diagnostic ability of DTS in detecting stent migration and obstruction was poorer than its ability to detect stent presence, meaning that DTS had limitations in detecting stent-related complications.

An airway stent is an essential tool for maintaining airway patency and relieving the symptoms of patients with benign or malignant airway disease (24). Previous studies reported that the overall stent-related complication rate was $23-34 \%$, and almost all patients required additional bronchoscopic intervention (25). Thus, it is important to monitor whether the stent is properly located and whether there are any stent-related complications. Our study is the first to evaluate the detection ability of DTS for silicone airway stents and stent-related complications in patients who have undergone bronchoscopic intervention. Choo et al. suggested that DTS is a more sensitive modality for detecting airway lesions than CXR (26). However, the study only judged the presence of airway lesions and did not target patients who received bronchoscopic intervention or had a silicone airway stent. 
Table 2 Diagnostic performance of CXR and DTS for silicone stent detection (N=213)

\begin{tabular}{|c|c|c|c|c|c|}
\hline Variables & \multicolumn{2}{|c|}{ CXR } & \multicolumn{2}{|c|}{ DTS } & $\mathrm{P}$ \\
\hline \multicolumn{6}{|l|}{ Total } \\
\hline Sensitivity\% & $119 / 167$ & $71.3(63.8-78.0)$ & $155 / 167$ & $92.8(87.8-96.2)$ & $<0.001$ \\
\hline Specificity\% & $46 / 46$ & $100.0(92.3-100.0)$ & $45 / 46$ & 97.8 (88.5-99.9) & 0.314 \\
\hline PPV\% & $119 / 119$ & 100.0 & $155 / 156$ & 99.4 (95.7-99.9) & 0.398 \\
\hline NPV\% & $46 / 94$ & $48.9(43.0-54.9)$ & $45 / 57$ & $79.0(68.5-86.6)$ & $<0.001$ \\
\hline \multicolumn{6}{|l|}{ Trachea* } \\
\hline Sensitivity\% & $41 / 64$ & $64.1(51.1-75.7)$ & $55 / 64$ & $85.9(75.0-93.4)$ & $<0.001$ \\
\hline PPV\% & $41 / 41$ & 100.0 & $55 / 56$ & $98.2(88.6-99.7)$ & 0.390 \\
\hline NPV\% & $147 / 170$ & 86.5 (82.2-89.9) & $146 / 155$ & 94.2 (89.9-96.8) & $<0.001$ \\
\hline \multicolumn{6}{|l|}{$\mathrm{RMB} \& \mathrm{RBI}{ }^{*}$} \\
\hline Sensitivity\% & $10 / 23$ & $43.5(23.2-65.5)$ & $21 / 23$ & 91.3 (72.0-98.9) & $<0.001$ \\
\hline Specificity\% & $188 / 188$ & $100.0(98.1-100.0)$ & $188 / 188$ & $100.0(98.1-100.0)$ & - \\
\hline Accuracy\% & $198 / 211$ & $93.8(89.7-96.7)$ & $209 / 211$ & 99.1 (96.6-99.9) & 0.003 \\
\hline PPV\% & $10 / 10$ & 100.0 & $21 / 21$ & 100.0 & - \\
\hline NPV\% & $188 / 201$ & 93.5 (91.0-95.4) & $188 / 190$ & $99.0(96.2-99.7)$ & 0.005 \\
\hline NPV\% & $133 / 145$ & 91.7 (86.8-94.9) & $133 / 134$ & 99.3 (95.0-99.9) & 0.003 \\
\hline
\end{tabular}

CXR and DTS images were evaluated using the bronchoscopic view as a reference, and all cases were evaluated as "Yes (Detected)" or "No (Not detected)". *, except for two Y-stent cases. CXR, chest radiography; DTS, digital tomosynthesis; Cl, confidence interval; PPV, positive predictive value; NPV, negative predictive value; RMB, right main bronchus; RBI, right bronchus intermedius; LMB, left main bronchus.

First, the diagnostic ability of DTS to detect stent presence was excellent, and its ability to evaluate stent migration or stent obstruction was also better than CXR. Regarding the detection of stent migration and obstruction due to granulation tissue or fibrosis with DTS, the sensitivity was approximately $45 \%$ and $65 \%$, respectively, which was lower than its ability to detect the presence of an airway stent. There is a possibility that an accurate comparison of stent migration data with the findings from bronchoscopic imaging was difficult due to the time interval between the procedure and the date of the imaging test in this retrospective study. Additionally, it is possible that the clinician was able to visualize the fine movement of the stent from the bronchoscopic view, but DTS was not able to detect such a fine migration. Granulation tissue and fibrosis might be difficult to detect by DTS because the density of these lesions is lower than that of silicon material stents. Nevertheless, stent-related complications such as migration, 


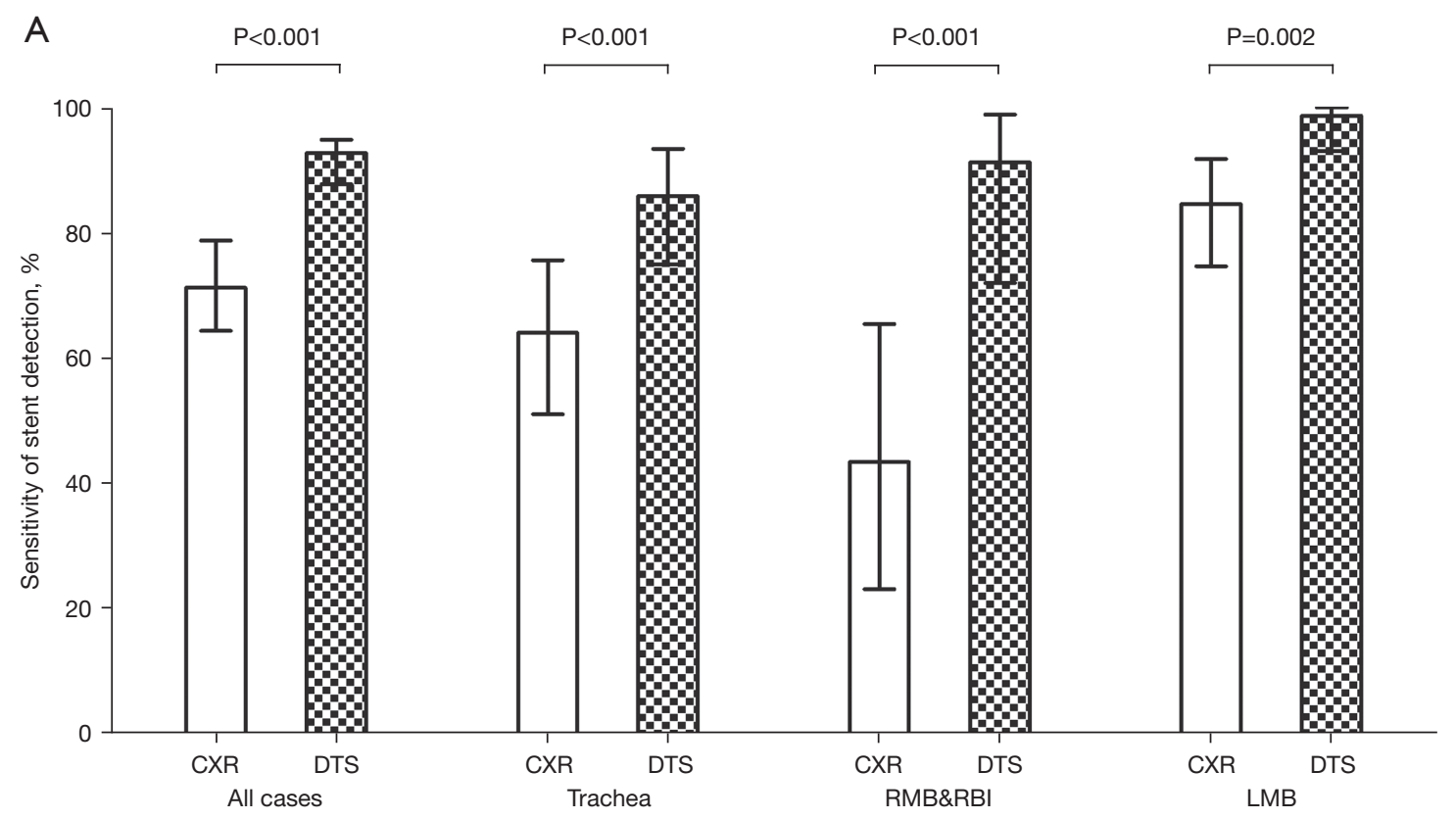

B

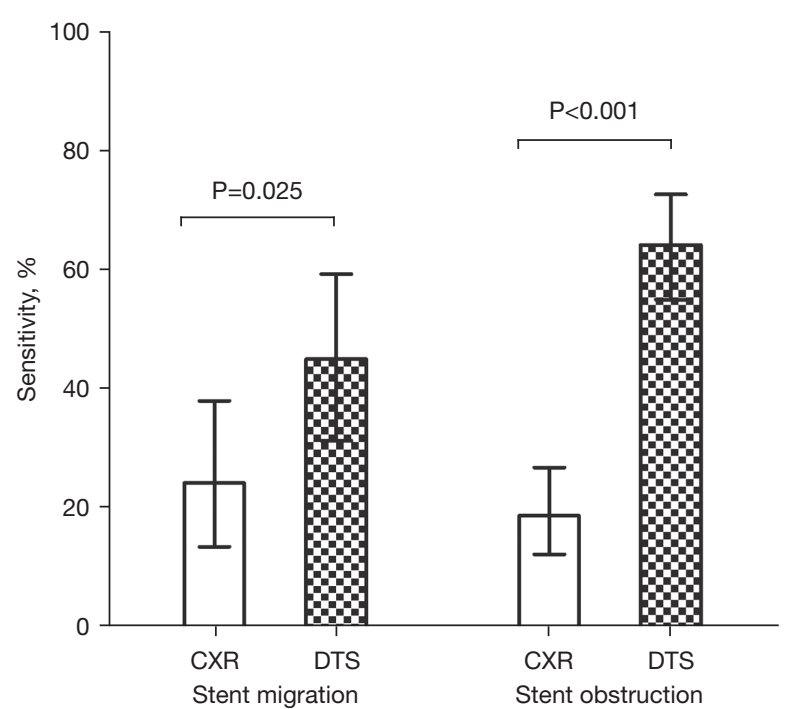

Figure 1 CXR and DTS sensitivity for (A) silicone airway stent detection and (B) stent migration and stent obstruction. Whiskers indicate 95\% confidence intervals for each sensitivity. CXR, chest radiography; DTS, digital tomosynthesis.

obstruction due to granulation tissue, and fibrosis are challenging to diagnose with CXR. Thus, DTS might be a better diagnostic tool than CXR for identifying stentrelated complications.

Previous studies suggested that chest CT is a relatively accurate and non-invasive method for diagnosing airway stenosis and stent-related complications $(27,28)$. However, patients with airway disease might need frequent follow- up and repeat procedures depending on their etiology, the presence of a stent, and the onset of stent-related complications. In our study, approximately $47 \%$ of the patients had two or more procedures. Stent-related complications can develop within the first 2 to 3 months after stent placement, and stent migration and granulation tissue formation may occur as early as 3 days later $(29,30)$. For this reason, patients who undergo bronchoscopic 
Table 3 Diagnostic performance for silicone stent-related complications of CXR and DTS (N=213)

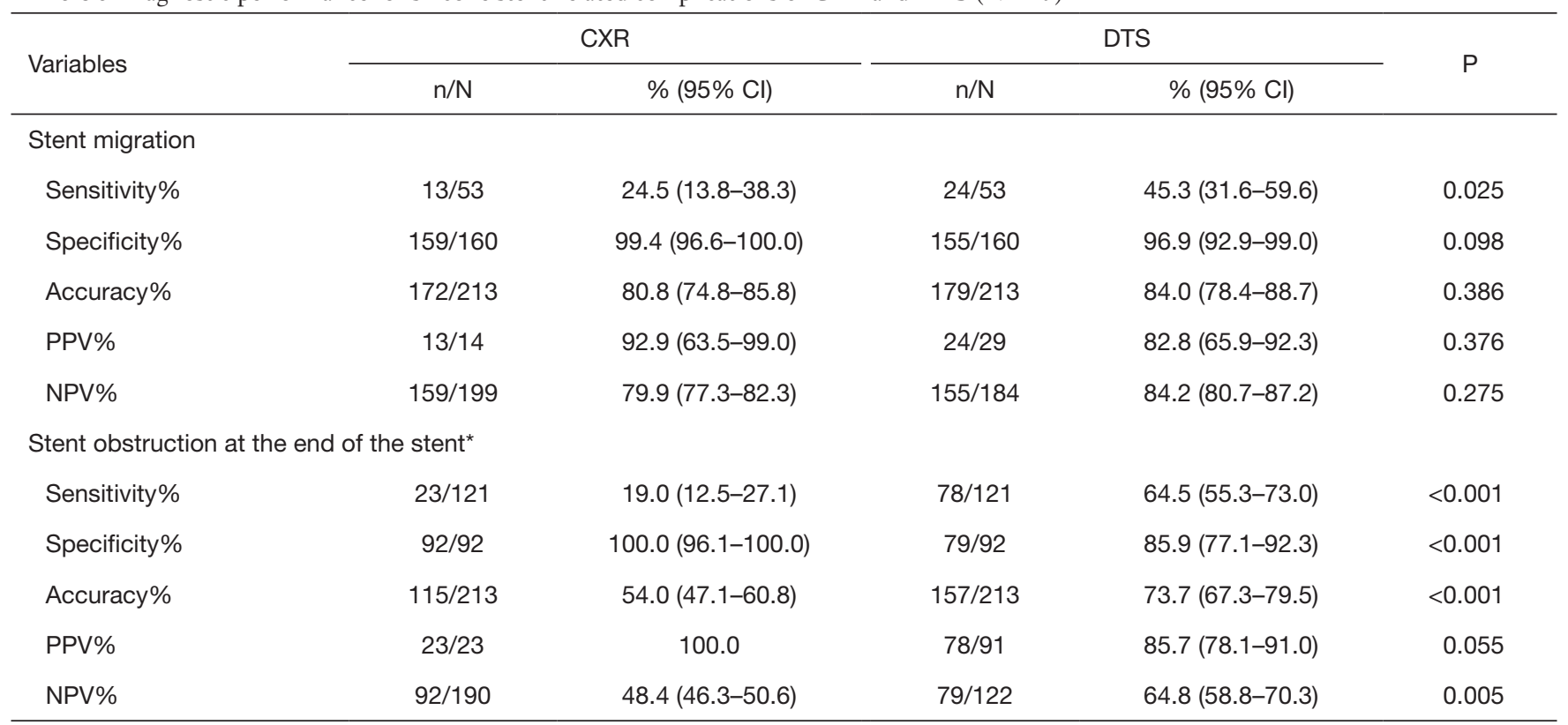

*, due to granulation tissue or fibrosis. CXR and DTS images were evaluated using the bronchoscopic view as a reference, and all cases were evaluated as "Yes (Detected)" or "No (Not detected)". CXR, chest radiography; DTS, digital tomosynthesis; Cl, confidence interval; PPV, positive predictive value; NPV, negative predictive value.
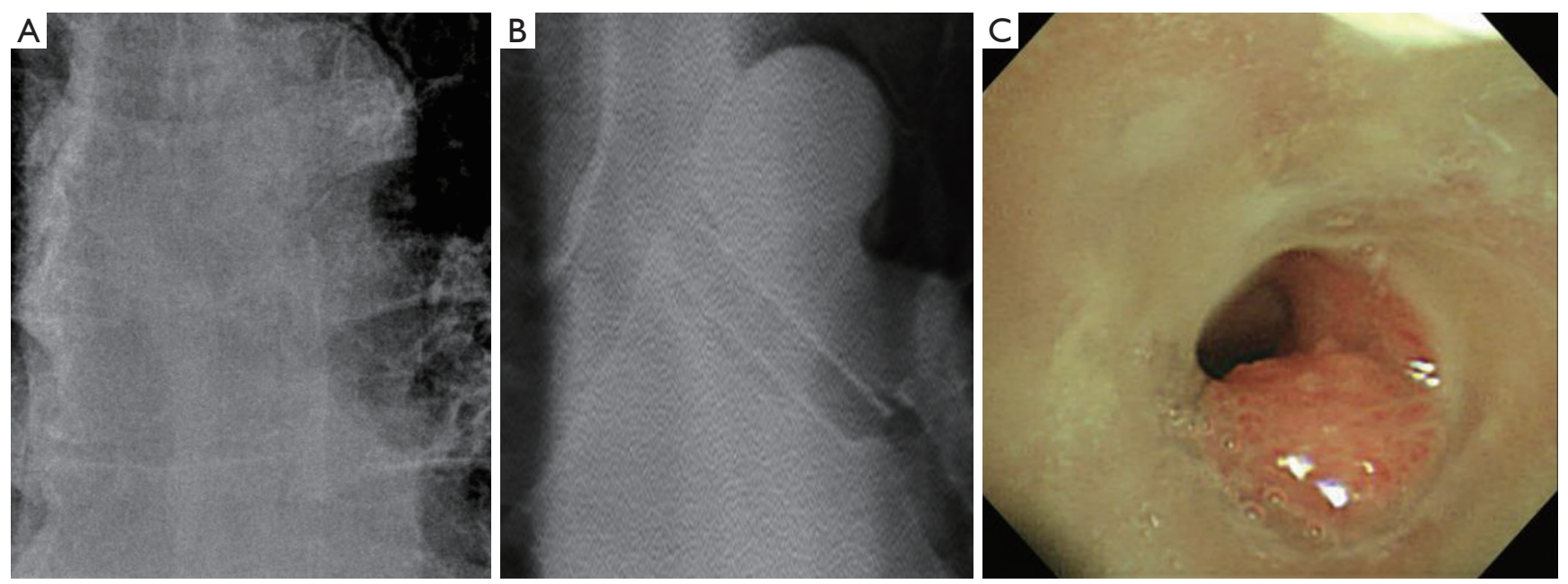

Figure 2 The airway stent was inserted into the LMB. The granulation tissue had grown at the distal end of stent. (A) On CXR, the stent and granulation tissue are hardly detected, but (B) on DTS, stent and airway lesion are detected well. (C) The actual appearance on bronchoscopic view. CXR, chest radiography; LMB, left main bronchus.

intervention might undergo imaging tests within a short period of time. In addition, elective stent removal is usually performed 6-18 months after stent insertion in patients with benign airway stenosis (31-33), and long-term followup is required. Therefore, when regular follow-up imaging tests are performed, the cumulative radiation dose should be considered in these patients. The effective dose of DTS $[0.05-0.2 \mathrm{mSv}(11,34,35)]$ is approximately 30 times lower than that of CT [1.5-8.0 mSv $(36,37)]$. In addition, CT is approximately three times more expensive than DTS based 

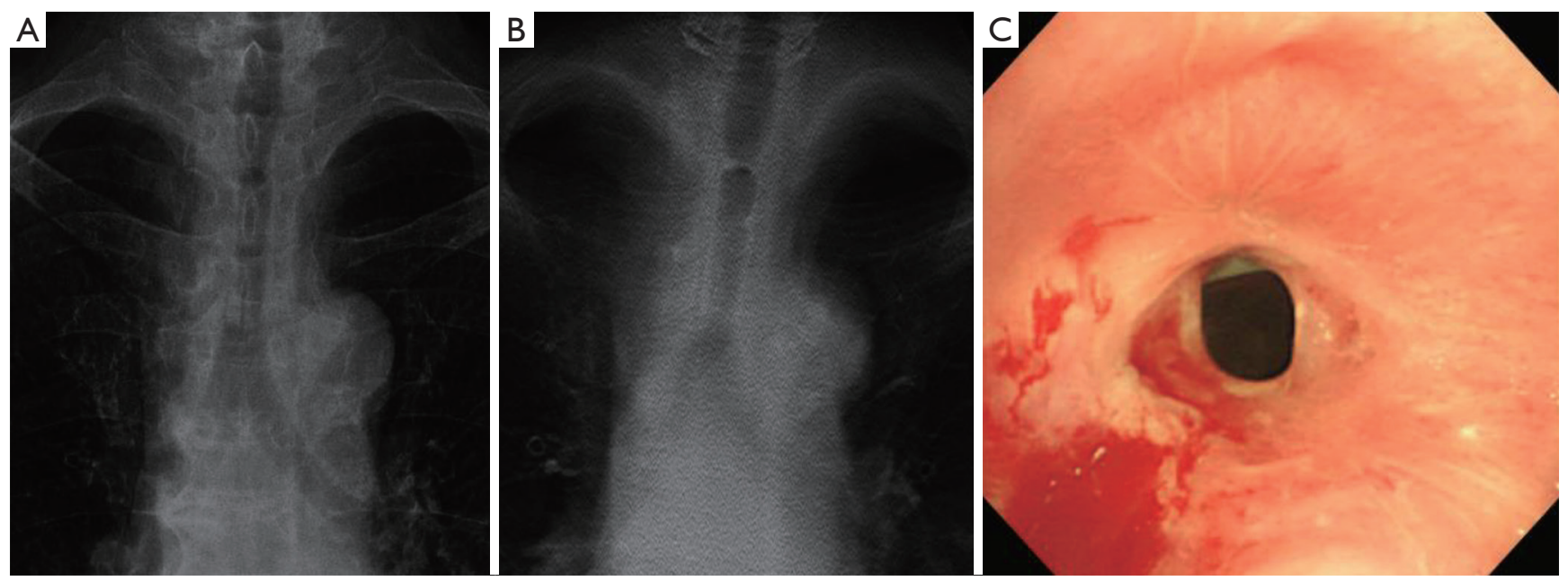

Figure 3 The airway stent was inserted into the mid-trachea, and fibrosis, creating an airway obstruction, occurred at the end of the stent. (A) On CXR, the stent and fibrosis were hardly detected. (C) On DTS, the trachea stent and fibrosis at the end are prominent. (C) The actual appearance on bronchoscopic view. CXR, chest radiography; DTS, digital tomosynthesis.

on Korean medical insurance standards. Thus, periodic follow-up with DTS may be a good alternative to CT in patients with airway disease, especially in patients with silicone airway stent insertion.

However, DTS has some limitations. The depth resolution of reconstructed cross-sectional images is one of the limitations of DTS because the projected images from DTS are not acquired at more than $360^{\circ}$ (38). Therefore, DTS cannot achieve the same isotropic resolution as chest CT. Another limitation of DTS is motion artifacts (16). Motion artifacts in DTS examinations can be relatively common because the images are collected within one breathhold. The DTS examination takes approximately $10 \mathrm{~s}$ to run once; therefore, breathing during image acquisition may result in severe artifacts in the section images. Patients with airway lesions and stent-related complications scheduled for bronchoscopic intervention might not hold their breath properly during the DTS examination due to severe dyspnea. Finally, DTS has the disadvantage of higher radiation exposure than CXR. CXR has a radiation exposure of approximately $0.02 \mathrm{mSv}$, but DTS has a higher radiation exposure than CXR. However, a method for testing with low-dose DTS has been developed (11). It also has a higher radiation exposure than CXR, but still a significantly lower radiation exposure than $\mathrm{CT}$.

Our institution routinely conducts DTS immediately after stent insertion, 1 month after, and 3 months after follow-up. After that, if the patient does not have any additional problems, we follow-up the patient's airway stent status with DTS every 6 months. However, in an early outpatient clinic visit with complaints of symptoms or an emergency room visit, the stent status is evaluated by DTS, chest CT, or flexible bronchoscopy, considering the patient's condition. Then a decision is made on bronchoscopic intervention. When DTS detects the presence or absence of stents for each location of the central airway, the ability to detect stents located in the trachea was the worst, and the ability to detect stents located in the LMB was the best. Some patients with tracheal lesions had an airway lesion or a stent in the upper third of the trachea. The silicone airway stent in that area was not covered well in the DTS images, and this might affect the diagnostic performance of DTS. Therefore, in our institution, in patients with airway stents in the upper trachea radiography and neck anteroposterior and lateral radiography are performed together with DTS.

This study has several limitations. First, this was a retrospective and single-center study; thus, there may have been a selection bias. Second, CXR, DTS, and bronchoscopic interventions were not performed on the same day; therefore, there was an interval between the procedure and imaging test. The time difference between imaging tests and procedures might cause a difference between the bronchoscopic view and DTS imaging of stentrelated complications, resulting in a low expected ability of DTS. However, CXR and DTS, the two imaging modalities compared in this study, had the same conditions because 
they were conducted simultaneously.

Despite these limitations, this is the first study to suggest that DTS can provide important information about silicone airway stents and stent-related complications to clinicians performing bronchoscopic intervention. Based on these results, we suggest that DTS is better than CXR for the follow-up examination of patients who have undergone bronchoscopic intervention. Further prospective studies are needed to evaluate the airway condition of patients who undergo stent insertion under bronchoscopic intervention through a prospective study.

In conclusion, DTS was more sensitive and accurate in detecting silicone airway stents and silicone stentrelated complications than CXR. However, there were limitations in confirming stent migration and obstruction due to granulation tissue growth and fibrosis in DTS. The diagnostic yield of DTS to detect stent-related complications was not satisfactory; however, considering the results of our study and the radiation dose administered to patients, DTS might be sufficient for regular long-term follow-up imaging tests compared to CXR or CT.

\section{Acknowledgments}

Funding: None.

\section{Footnote}

Reporting Checklist: The authors have completed the TREND reporting checklist. Available at https://dx.doi. org/10.21037/jtd-21-1032

Data Sharing Statement: Available at https://dx.doi. org/10.21037/jtd-21-1032

Peer Review File: Available at https://dx.doi.org/10.21037/ jtd-21-1032

Conflicts of Interest: All authors have completed the ICMJE uniform disclosure form (available at https://dx.doi. org/10.21037/jtd-21-1032). The authors have no conflicts of interest to declare.

Ethical Statement: The authors are accountable for all aspects of the work in ensuring that questions related to the accuracy or integrity of any part of the work are appropriately investigated and resolved. This study was approved by the Samsung Medical Center Institutional
Review Board (IRB No.2020-12-067), and informed consent was waived as we only used de-identified patients' information, and this study was a retrospective study. The study was conducted in accordance with the Declaration of Helsinki (as revised in 2013).

Open Access Statement: This is an Open Access article distributed in accordance with the Creative Commons Attribution-NonCommercial-NoDerivs 4.0 International License (CC BY-NC-ND 4.0), which permits the noncommercial replication and distribution of the article with the strict proviso that no changes or edits are made and the original work is properly cited (including links to both the formal publication through the relevant DOI and the license). See: https://creativecommons.org/licenses/by-nc-nd/4.0/.

\section{References}

1. McAdams HP, Samei E, Dobbins J 3rd, et al. Recent advances in chest radiography. Radiology 2006;241:663-83.

2. Raoof S, Feigin D, Sung A, et al. Interpretation of plain chest roentgenogram. Chest 2012;141:545-58.

3. Kwong JS, Adler BD, Padley SP, et al. Diagnosis of diseases of the trachea and main bronchi: chest radiography vs CT. AJR Am J Roentgenol 1993;161:519-22.

4. Raju S, Ghosh S, Mehta AC. Chest CT Signs in Pulmonary Disease: A Pictorial Review. Chest 2017;151:1356-74.

5. Dobbins JT 3rd, McAdams HP. Chest tomosynthesis: technical principles and clinical update. Eur J Radiol 2009;72:244-51.

6. Dobbins JT 3rd, McAdams HP, Godfrey DJ, et al. Digital tomosynthesis of the chest. J Thorac Imaging 2008;23:86-92.

7. Quaia E, Baratella E, Cernic S, et al. Analysis of the impact of digital tomosynthesis on the radiological investigation of patients with suspected pulmonary lesions on chest radiography. Eur Radiol 2012;22:1912-22.

8. Quaia E, Baratella E, Cioffi V, et al. The value of digital tomosynthesis in the diagnosis of suspected pulmonary lesions on chest radiography: analysis of diagnostic accuracy and confidence. Acad Radiol 2010;17:1267-74.

9. Vikgren J, Zachrisson S, Svalkvist A, et al. Comparison of chest tomosynthesis and chest radiography for detection of pulmonary nodules: human observer study of clinical cases. Radiology 2008;249:1034-41.

10. Dobbins JT 3rd, McAdams HP, Sabol JM, et al. MultiInstitutional Evaluation of Digital Tomosynthesis, 
Dual-Energy Radiography, and Conventional Chest Radiography for the Detection and Management of Pulmonary Nodules. Radiology 2017;282:236-50.

11. Kim EY, Chung MJ, Lee HY, et al. Pulmonary mycobacterial disease: diagnostic performance of low-dose digital tomosynthesis as compared with chest radiography. Radiology 2010;257:269-77.

12. Meltzer C, Vikgren J, Bergman B, et al. Detection and Characterization of Solid Pulmonary Nodules at Digital Chest Tomosynthesis: Data from a Cohort of the Pilot Swedish Cardiopulmonary Bioimage Study. Radiology 2018;287:1018-27.

13. Quaia E, Baratella E, Poillucci G, et al. Diagnostic impact of digital tomosynthesis in oncologic patients with suspected pulmonary lesions on chest radiography. Eur Radiol 2016;26:2837-44.

14. Kim JH, Lee KH, Kim KT, et al. Comparison of digital tomosynthesis and chest radiography for the detection of pulmonary nodules: systematic review and meta-analysis. Br J Radiol 2016;89:20160421.

15. Zakaluzny SA, Lane JD, Mair EA. Complications of tracheobronchial airway stents. Otolaryngol Head Neck Surg 2003;128:478-88.

16. Kim SM, Chung MJ, Lee KS, et al. Digital tomosynthesis of the thorax: the influence of respiratory motion artifacts on lung nodule detection. Acta Radiol 2013;54:634-9.

17. Shin B, Chang B, Kim H, et al. Interventional bronchoscopy in malignant central airway obstruction by extra-pulmonary malignancy. BMC Pulm Med 2018;18:46.

18. Kim BG, Lee K, Um SW, et al. Clinical outcomes and the role of bronchoscopic intervention in patients with primary pulmonary salivary gland-type tumors. Lung Cancer 2020;146:58-65.

19. Tay CK, Jeong BH, Kim H. Angulated Stents-A Novel Stent Improvisation to Manage Difficult Post-tuberculosis Bronchial Stenosis. ASAIO J 2018;64:565-9.

20. Trevethan R. Sensitivity, Specificity, and Predictive Values: Foundations, Pliabilities, and Pitfalls in Research and Practice. Front Public Health 2017;5:307.

21. Mercaldo ND, Lau KF, Zhou XH. Confidence intervals for predictive values with an emphasis to case-control studies. Stat Med 2007;26:2170-83.

22. Campbell I. Chi-squared and Fisher-Irwin tests of two-bytwo tables with small sample recommendations. Stat Med 2007;26:3661-75.

23. Richardson JT. The analysis of $2 \times 2$ contingency tables-yet again. Stat Med 2011;30:890; author reply 891-2.
24. Guibert N, Saka H, Dutau H. Airway stenting: Technological advancements and its role in interventional pulmonology. Respirology 2020;25:953-62.

25. Murgu SD, Egressy K, Laxmanan B, et al. Central Airway Obstruction: Benign Strictures, Tracheobronchomalacia, and Malignancy-related Obstruction. Chest 2016;150:426-41.

26. Choo JY, Lee KY, Yu A, et al. A comparison of digital tomosynthesis and chest radiography in evaluating airway lesions using computed tomography as a reference. Eur Radiol 2016;26:3147-54.

27. Ferretti GR, Knoplioch J, Bricault I, et al. Central airway stenoses: preliminary results of spiral-CT-generated virtual bronchoscopy simulations in 29 patients. Eur Radiol 1997;7:854-9.

28. Ferretti GR, Kocier M, Calaque O, et al. Follow-up after stent insertion in the tracheobronchial tree: role of helical computed tomography in comparison with fiberoptic bronchoscopy. Eur Radiol 2003;13:1172-8.

29. Inchingolo R, Sabharwal T, Spiliopoulos S, et al. Tracheobronchial stenting for malignant airway disease: long-term outcomes from a single-center study. Am J Hosp Palliat Care 2013;30:683-9.

30. Lee HJ, Labaki W, Yu DH, et al. Airway stent complications: the role of follow-up bronchoscopy as a surveillance method. J Thorac Dis 2017;9:4651-9.

31. Eom JS, Kim H, Park HY, et al. Timing of silicone stent removal in patients with post-tuberculosis bronchial stenosis. Ann Thorac Med 2013;8:218-23.

32. Ryu YJ, Kim H, Yu CM, et al. Use of silicone stents for the management of post-tuberculosis tracheobronchial stenosis. Eur Respir J 2006;28:1029-35.

33. Martinez-Ballarin JI, Diaz-Jimenez JP, Castro MJ, et al. Silicone stents in the management of benign tracheobronchial stenoses. Tolerance and early results in 63 patients. Chest 1996;109:626-9.

34. Båth M, Svalkvist A, von Wrangel A, et al. Effective dose to patients from chest examinations with tomosynthesis. Radiat Prot Dosimetry 2010;139:153-8.

35. Yamada Y, Jinzaki M, Hasegawa I, et al. Fast scanning tomosynthesis for the detection of pulmonary nodules: diagnostic performance compared with chest radiography, using multidetector-row computed tomography as the reference. Invest Radiol 2011;46:471-7.

36. Silva AC, Lawder HJ, Hara A, et al. Innovations in CT dose reduction strategy: application of the adaptive statistical iterative reconstruction algorithm. AJR Am J 
Roentgenol 2010;194:191-9.

37. National Lung Screening Trial Research Team; Aberle DR, Adams AM, et al. Reduced lung-cancer mortality with low-dose computed tomographic screening. N Engl J Med 2011;365:395-409.

Cite this article as: Kim BG, Chung MJ, Jeong BH, Kim H. Diagnostic performance of digital tomosynthesis to evaluate silicone airway stents and related complications. J Thorac Dis 2021;13(10):5627-5637. doi: 10.21037/jtd-21-1032
38. Asplund S, Johnsson AA, Vikgren J, et al. Learning aspects and potential pitfalls regarding detection of pulmonary nodules in chest tomosynthesis and proposed related quality criteria. Acta Radiol 2011;52:503-12. 


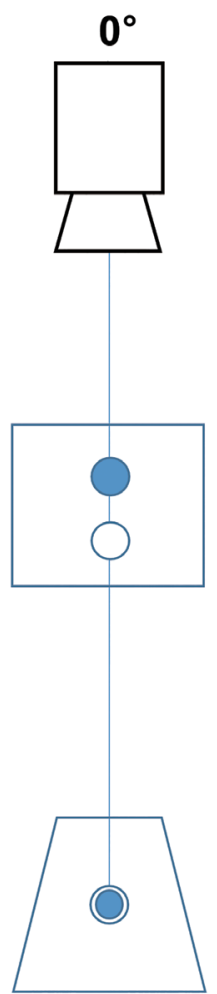

Plain chest radiography

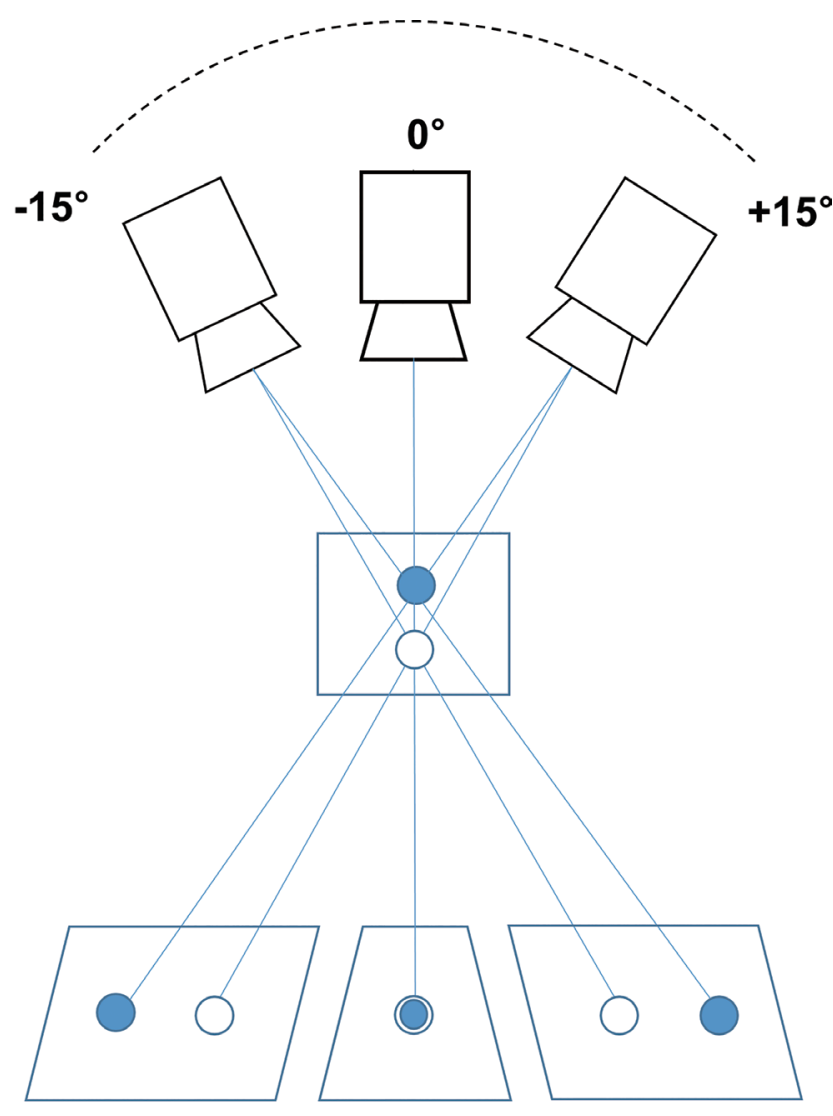

Digital tomosynthesis

Figure S1 Principle of CXR and DTS image acquisition. CXR, chest radiography; DTS, digital tomosynthesis.

Table S1 Diagnostic performance of CXR and DTS in visualizing silicone stent angulation (N=213)

\begin{tabular}{|c|c|c|c|c|c|}
\hline Variables & \multicolumn{2}{|c|}{ CXR } & \multicolumn{2}{|c|}{ DTS } & $P$ value \\
\hline \multicolumn{6}{|l|}{ Stent angulation } \\
\hline Sensitivity\% & $10 / 39$ & $25.6(13.0-42.1)$ & $26 / 39$ & $66.7(49.8-80.9)$ & $<0.001$ \\
\hline Specificity\% & $173 / 174$ & $99.4(96.8-100.0)$ & $167 / 174$ & $96.0(91.9-98.4)$ & 0.035 \\
\hline PPV\% & $10 / 11$ & 90.9 (56.9-98.7) & $26 / 33$ & $78.8(63.5-88.8)$ & 0.373 \\
\hline NPV\% & $173 / 202$ & $85.6(93.2-87.8)$ & $167 / 180$ & $92.8(85.9-94.2)$ & 0.025 \\
\hline
\end{tabular}

*, CXR and DTS images were evaluated using the bronchoscopic view as the reference, and all cases were evaluated as "Yes (Detected)" or "No (Not detected)". CXR, chest radiography; DTS, digital tomosynthesis; CI, confidence interval; PPV, positive predictive value; NPV, negative predictive value. 

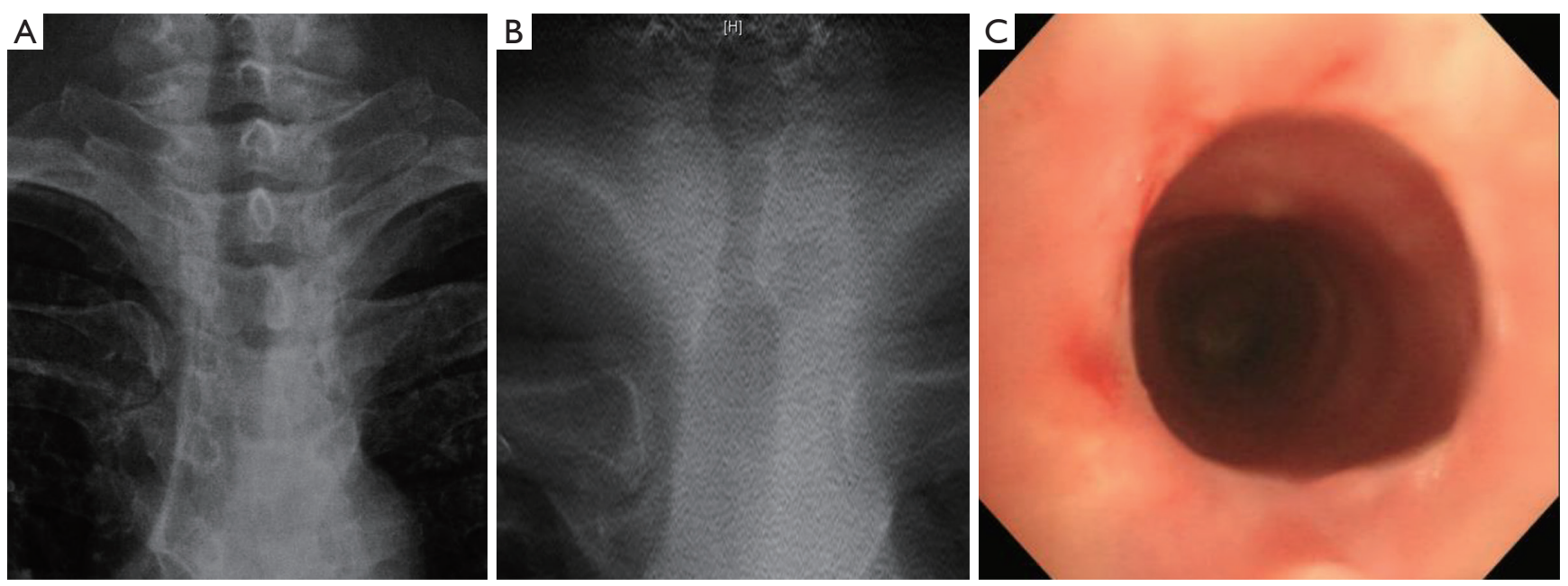

Figure S2 This is a case where the stent appears to be inserted into the trachea where the stent was previously present even though there is no actual airway stent. (A) Chest radiograph. (B) Digital tomosynthesis image. (C) The actual appearance in the bronchoscopic view. 
Table S2 A $2 \times 2$ contingency table according to each evaluation item $(\mathrm{N}=213)$

Detection in rigid bronchoscopy

Presence of silicone stent

Detection on CXR

$\begin{array}{ccccc} & \text { Yes } & \text { No } & \text { Total } & \text { Detection on DTS } \\ \text { Yes } & 119 & 0 & 119 & \\ \text { No } & 48 & 46 & 94 & \\ \text { Total } & 167 & 46 & 213\end{array}$

Presence of silicone stent at trachea

Detection on CXR

$\begin{array}{cccc} & \text { Yes } & \text { No } & \text { Total } \\ \text { Yes } & 41 & 0 & 41 \\ \text { No } & 23 & 147 & 170 \\ \text { Total } & 64 & 147 & 211\end{array}$

Detection on DTS

$\begin{array}{cccc} & \text { Yes } & \text { No } & \text { Total } \\ \text { Yes } & 155 & 1 & 156 \\ \text { No } & 12 & 45 & 57 \\ \text { Total } & 167 & 46 & 213\end{array}$

Presence of silicone stent at RMB \& RBI

Detection on CXR

$\begin{array}{cccc} & \text { Yes } & \text { No } & \text { Total } \\ \text { Yes } & 10 & 0 & 10 \\ \text { No } & 13 & 188 & 201 \\ \text { Total } & 23 & 188 & 211\end{array}$

Presence of silicone stent at LMB

Detection on CXR

$\begin{array}{cccc} & \text { Yes } & \text { No } & \text { Total } \\ \text { Yes } & 66 & 0 & 66 \\ \text { No } & 12 & 133 & 145 \\ \text { Total } & 78 & 133 & 211\end{array}$

Detection on DTS

$\begin{array}{cccc} & \text { Yes } & \text { No } & \text { Total } \\ \text { Yes } & 55 & 1 & 56 \\ \text { No } & 9 & 146 & 155 \\ \text { Total } & 64 & 147 & 211\end{array}$

Stent migration

Detection on CXR

$\begin{array}{ccccc} & \text { Yes } & \text { No } & \text { Total } & \text { Detection on DTS } \\ \text { Yes } & 13 & 1 & 14 & \\ \text { No } & 40 & 159 & 199 & \\ \text { Total } & 53 & 160 & 213\end{array}$

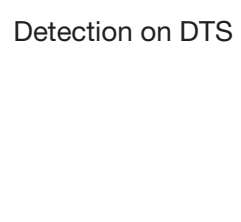

$\begin{array}{cccc} & \text { Yes } & \text { No } & \text { Total } \\ \text { Yes } & 21 & 0 & 21 \\ \text { No } & 2 & 188 & 190 \\ \text { Total } & 23 & 188 & 211\end{array}$

Detection on CXR

(n)

Stent obstruction

Detection on CXR

$\begin{array}{cccc} & \text { Yes } & \text { No } & \text { Total } \\ \text { Yes } & 23 & 0 & 23 \\ \text { No } & 98 & 92 & 190 \\ \text { Total } & 121 & 92 & 213\end{array}$

Detection on DTS

$\begin{array}{cccc} & \text { Yes } & \text { No } & \text { Total } \\ \text { Yes } & 78 & 13 & 91 \\ \text { No } & 43 & 79 & 122 \\ \text { Total } & 121 & 92 & 213\end{array}$

Stent angulation

Detection on CXR

$\begin{array}{cccc} & \text { Yes } & \text { No } & \text { Total } \\ \text { Yes } & 10 & 1 & 11 \\ \text { No } & 29 & 173 & 202 \\ \text { Total } & 39 & 174 & 213\end{array}$

Detection on DTS

$\begin{array}{cccc} & \text { Yes } & \text { No } & \text { Total } \\ \text { Yes } & 26 & 7 & 33 \\ \text { No } & 13 & 167 & 180 \\ \text { Total } & 39 & 174 & 213\end{array}$

CXR, chest radiography; DTS, digital tomosynthesis; RMB, right main bronchus; RBI, right bronchus intermedius; LMB, left main bronchus. 